\title{
Importing Space-time Concepts Into AGI
}

\author{
Eugene J. Surowitz \\ New York University (visiting) \\ PO Box 7639, Greenwich, CT 06836 \\ surow@attglobal.net
}

\begin{abstract}
Feedback cycles are proposed as the general unit of intellect and intelligence. This enables the importation of space-time concepts from physics. The action-lens, a processing structure based on these measurable objects, is defined. Larger assemblies of this structure may support the Lowen model of information processing by the brain.
\end{abstract}

\section{Cycles as the Fundamental State}

The purpose of this paper is to provide part of the rationale for adopting feedback loops as the particular dynamical structure fundamental for the construction of intelligent systems. This question was raised with respect to a paper submitted to AGI-08. What favors the adoption of processor cycles as a primitive unit of intellect?

Predicting the structure and behavior of large assemblies of processors or neurons, without an analyzable and controllable generation mechanism appears to create a combinatoric scaling barrier to creating of an AGI from those base units. This is very analogous to the problem of discovering the existence, structure, and properties of DNA molecules by examining every possible combination of elements from the periodic table. It would seem desirable to have an engineering approach instead.

Random graph theory predicts the occurrence of cycles when the number of connections equals the number of nodes. The neurons of the brain far exceed this criteria and we may assume that cycle structures are common. Cycles can be counted, at least in principle, and the number of cycles provides a measure of the internal structure of a system; fluctuations in that count can be a measure of dynamical properties of the system.

Cycles are less primitive than neurons or processors. An appropriate analogy would be the difference between atomic physics and chemistry. They are also recursively constructive as they can be inter-connected and will then inter-communicate to form larger cycle based structures. They then take on the aspect of primitive sub-assemblies, or molecules in the chemistry analogy. Adopting cycles of processors as a fundamental structure may be an effective way of addressing the scaling problem for AGI by reducing branching ratios as a prospective AGI system is enlarged.

Cycles create and define time-scales: the propagation time it takes for a signal's effects to completely travel the cycle's circumference and arrive at the originating node creates a clock based on this propagation time and defines a time-scale associated with the cycle. The cycle based time-scales enable the importation of spacetime concepts from physics. Those concepts, in turn, bootstrap the construction of processor structures to be used as engineering elements to generate still larger structures.

A by-product of that construction is the ability to support multiple short and long time-scales with a single primitive structure. This addresses the time-scale dichotomy of the need of organisms and systems to act in the short-term on plans with long-term objectives. In the context of AGI, the cycle time-scales define persistent objects competing for resources leading to action toward short and long term objectives. Groups of cycles of similar circumference will define a range of times for such actions. This suggests a conceptual structure, borrowed from physics, that is useful to draw and visualize cycle structures and bootstrap our ability to examine the space-time behavior of large numbers of co-located cycles.

\section{Cycle Space-Time and the Action-Lens}

Let's proceed with constructing a diagram showing the relationship of cycles and time: The set of cycles with all possible circumferences forms an infinite disk when laid out as circles on a plane with a common center. (Here, we are assuming large numbers of nodes in each cycle which creates an image of continuity.) This is the "space" portion of the space-time we are constructing. Plot the respective cycle-times on a vertical axis thru the common center. This is the "time" portion of the space-time.

This visual aid suggests a structure combining a large base cycle, which processes over extended physical times, with a much smaller focus structure, even a single node, which works on much shorter time-scales, into a single object to be used as a building block to 
construct systems. This structure will be called the "action-lens" in the sequel.

The nodes of a cycle of processors, that operates over multiples of its local cycle-time, are a persistent object, the base-cycle. Again, visualize a cone of connectivity with its base coincident with a cycle and its apex on the cycle-time axis at the point corresponding to the cycle-time of our particular cycle. The visualized cone itself would be the surface created by considering the base-cycle so dense in processor nodes as to be almost continuous. In general the connectivity of the base-cycle need not be so great. The cycle-times of cycles with similar circumference land in a small but not necessarily contiguous neighborhood on the cycle-time axis. The cycle-time axis can now be thought of as a linear array of nodes with each successive node situated at a slightly greater time along the axis.

The fraction of the nodes of a base-cycle directly linked to a single node, the focus-node, looks like a tepee's poles without the shell. More generally, the focus-node can be replaced by a set of nodes that are almost completely connected, to some approximation of completeness in the graph theoretical sense. This also allows similar circumference cycles to feed a set of focus-nodes, with very rapid propagation of signals among the focus-nodes, relative to the base cycle-times. What does the focus-node/graph do? The focus-node or graph provides a second time-scale for the base-cycle. It creates short term effects from a persistent object whose existence is measured only on larger cycle-time scales. One might consider the activity of assembling a model from a kit of parts as quick part-steps carried out to yield the final model, the long-term objective.

The combination of base-cycles and a focus-graph constitute the "action-lens". The term "lens" is borrowed from optics in order to cover more elaborate versions of this object suggested by the space-time construction. The action-lens is intended to replace the halt-free Turing machines used at the lowest level of cycle constructs. Rather than use arbitrary or random inter-connections of nodes, we can use the action-lens as an engineering object to construct larger structures.

\section{Mesoscale Structure}

The mesoscale, or middle scale of structure, between primitive processors and the macroscopic scale of general intelligence functioning may be approachable if we use recursive definition on the action-lens structure. As an example, we could replace the elementary processors of the basic action-lens with the action-lens structure itself thus generating an action-lens of action-lenses.

Under the control of executive functions, we could generate other action-lens based complexes. As examples, these could connect a lens's base-cycle to other lens's base-cycles, connect the focus-graph to other foci, and connect foci to base-cycle nodes. Respectively, these yield a stack structure, an hour-glass, and a multilayer. Gluing action-lenses at nodes along their base- cycles gives the stack; gluing focus to focus creates the hour-glass; gluing an inverted layer of action-lenses to an upright layer of action-lenses, creates a bilayer.

Unconstrained generation at the mesoscale could, and probably would, lead to a space of generated functions so large that determining those most useful for AGI applications would be difficult at best. A macroscopic model that provides a candidate set of target functions to be generated by mesoscale generation operators would be one possible way of imposing constraints somewhat in the manner of a variational problem. There is such a candidate model, the Lowen model, which serves to supply the target high-level functions.

\section{The Lowen Model of Consciousness}

On the macroscopic scale, Walter Lowen, late of the State University of New York at Binghamton, constructed a model of the conscious processes of the mind (Lowen 1982), inspired by Carl Jung's theory of psychological types. Lowen founded his concepts on information processing and system theory.

The Lowen information processing model conceives of consciousness as constructed from specialized processes, variously termed capacities or poles in the model. Sensory data is taken and handed from capacity to capacity while eventually producing behavioral outputs. Some of these capacities such as sorting, path finding, and decision tree processing are identifiable as well studied topics of computer science. The list of capacities was limited to a set of sixteen which arose from an analysis of the Jungian classification personality types.

A basic result of the Lowen model is the discovery of two macroscopic processing circuits, in essence, cycles, among the capacities. These circuits weakly, but importantly, communicate with each other to create the response patterns we call personality types. If further support for their existence is found by or in psychological research, the presence of such macroscopic circuits would be a further argument for using the Lowen model as one end point of what amounts to a variational problem: How to construct the unconscious mesoscale, starting with a mass of primitive processors and ending with the preconscious capacities.

The suggestion that the action-lens structure constitutes a useful basis for the construction of Lowenstyle AGI systems is being explored in these ways: The Lowen capacities do not all have identifications with established information processing technologies; such identifications need to be found and formalized. Can the action-lens model be used to generate larger structures that act as the classical specialized information processors of the Lowen model? Is it practical to use recursive function theory to generate and control the development of large scale structures effectively?

\section{References}

Lowen, W. 1982. Dichotomies of the Mind. New York, NY: John Wiley. 